\title{
28 Research Square \\ miR-221-3p Promotes Pancreatic Cancer Progression via Targeting P53 and PTEN
}

\author{
Jian-Jun Wu \\ the Second Hospital of Shanxi Medical University \\ Zhong-Hua Shang ( $\nabla$ zhshang_sxmu@163.com) \\ the Second Hospital of Shanxi Medical University \\ Min Guo \\ the Second Hospital of Shanxi Medical University \\ Zhen-Hua Yang \\ the Second Hospital of Shanxi Medical University

\section{Fang-Gang Ren} \\ the Second Hospital of Shanxi Medical University

\section{Si-Cheng Bian} \\ the Second Hospital of Shanxi Medical University
}

\section{Research Article}

Keywords: pancreatic cancer, miR-221-3p, P53, PTEN, proliferation, migration, invasion, apoptosis

Posted Date: December 13th, 2021

DOI: https://doi.org/10.21203/rs.3.rs-1156414/v1

License: (c) (i) This work is licensed under a Creative Commons Attribution 4.0 International License. Read Full License 


\section{Abstract}

Background: To assess the regulatory role of microRNA-221-3p (miR-221-3p) as a modulator of pancreatic cancer (PC) cell apoptosis, invasion, migration, and proliferation through its ability to regulate P53 and PTEN expression.

Methods: PCPATU8988 cells were used as a model in which high-level or low-level models of miR-221-3p expression were established, with qPCR being used to confirm transfection efficiency. CCK-8 assays were employed to evaluate the proliferation of these cells, with migration and invasion being assessed through appropriate in vitro assays. Western blotting was used to assess PTEN and P53 protein levels in these experimental cells. Flow cytometry was additionally used to assess the impact of experimental manipulations on cellular apoptosis.

Results: MiR-221-3p overexpression enhanced the migratory, proliferative, and invasive activity of PCPATU8988 cells $(P<0.01)$ and suppressed their apoptotic death $(P<0.05)$, while miR-221-3p inhibition had the opposite impact. No differences in P53 and PTEN protein levels were detected when comparing the NC miR-221-3p mimic or inhibitor groups ( $P>0.05)$, whereas miR-221-3p mimic transfection significantly reduced the levels of these proteins $(P<0.05)$.

Conclusion: This analysis showed that miR-221-3p can drive PC cell proliferative, migratory, and invasive activity while suppressing the apoptotic death of these cells. Functionally, this miRNA can suppress P53 and PTEN protein expression. Overall, this suggests that miR-221-3p can regulate PC development by controlling the expression of these key oncogenic proteins.

\section{Introduction}

Pancreatic cancer (PC) is highly malignant and often insidious in onset, resulting in a poor patient prognosis that necessitates an early diagnosis to ensure optimal patient outcomes. Over $85 \%$ of PC patients exhibit advanced -stage PC at the time of initial diagnosis, and are thus unable to undergo radical curative surgical treatment. Without treatment, these patients exhibit a median 5-8 month survival period and a $<5 \%$ five-year survival rate. ${ }^{1} \mathrm{PC}$ tumor cells exhibit a range of highly malignant properties including abnormal proliferative activity, a high potential for metastasis, and invasivity that make them well-suited to rapid disease progression. ${ }^{2}$ However, further research is necessary to fully clarify the molecular mechanisms underlying PC cell malignancy.

MicroRNAs (miRNAs) are short ( 22 nucleotide) post-transcriptional regulators of gene expression without coding potential. ${ }^{3}$ Many miRNAs can shape tumorigenesis and associated processes, or can offer value in the context of cancer patient diagnosis, prognostic evaluation, and treatment. ${ }^{4-6}$ Specific miRNAs function by regulating tumor cell differentiation, survival, proliferation, and metastasis through their ability to control the expression of specific target genes. ${ }^{7}$ 
Prior work has identified miR-221-3p as an anti-angiogenic miRNA encoded within an X chromosome gene cluster that is expressed at high levels within human atherosclerotic vascular endothelial cells of the intimal layer. ${ }^{8}$ In several cancers, miR-221-3p has also been shown to regulate abnormal cellular proliferation and differentiation, ${ }^{9-12}$ although only a few studies have examined it in the context of PC. ${ }^{13-}$ ${ }^{15}$ In vitro, there is evidence that miR-221-3p can promote PC cell proliferation, ${ }^{16}$ whereas Sarkar et al. found that it was able to suppress PC cell proliferation by promoting the upregulation of PUMA and other downstream proteins. ${ }^{17}$ In triple-negative breast cancer patients, low levels of miR-221-3p expression have been linked to a poor prognosis through a mechanism linked with its regulation of PARP $1 .{ }^{18}$ In most solid tumors, however, miR-221-3p upregulation is commonly observed, and the mechanistic basis for such upregulation remains unclear. There is thus a clear need for further studies of the specific target genes of this miRNA in PC and for the identification of effective treatments centered around this key genetic regulator.

The transcription facto P53 plays an essential role in maintaining genomic integrity, monitoring for abnormalities that arise during DNA replication and cell cycle progression, inducing repair gene upregulation when errors are detected, and promoting apoptotic cell death when they are not repaired, thereby eliminating cells with the potential to undergo oncogenic transformation. Owing to its potent tumor suppressor activity, P53 is frequently inactivated in tumors through deletion or inactivation, thereby enhancing the proliferative activity of cancer cells and decreasing their susceptibility to apoptotic death. ${ }^{19} \mathrm{~A}$ range of translation and post-translational modifications control P53 expression and function, including phosphorylation, ubiquitination, and proteasomal degradation. Wild-type (WT) P53 expression is observed in roughly half of human tumors, and in these cases, the suppression or inactivation of P53 negative regulators can prove an effective therapeutic intervention. ${ }^{20}$ For example, the accumulation of P53 and MDM2 overexpression within hepatocellular carcinoma tumors is negatively correlated with patient survival. ${ }^{21}$ In chondrocytes, MDM2 has been established as a miR-221-3p target. ${ }^{22}$ PTEN is another critically important tumor suppressor gene in many cancers that is encoded on chromosome 10q23.31. ${ }^{23,24}$ PTEN regulates protein phosphatase and 3'-phosphoinositol phosphatase functionality, dephosphorylating the second messenger in the PI3K pathway, phosphatidylinositol 3,4,5-triphosphate (PIP3), thereby inhibiting the activity of AKT family serine/threonine kinases, which are important serine/threonine protein kinases. ${ }^{25,26}$ AKT activity regulates the proliferative activity, invasive potential, and apoptotic death of many tumors. ${ }^{27}$ In tumors exhibiting PTEN inactivation, AKT overactivation is observed, resulting in reduced apoptotic tumor cell death and enhanced proliferative activity. ${ }^{28,29} \mathrm{In}$ gastrointestinal cancer, miR-221-3p has been suggested to promote enhanced tumor cell apoptosis via KIT/AKT signaling pathway regulation. ${ }^{30}$

In light of the above evidence, this study was formulated with the goal of clarifying the role of miR-221-3p as a regulator of the survival, migration, proliferation, and invasion of PC cells. Through a series of in vitro analyses, P53 and PTEN were validated as miR-221-3p target genes in PC tumors, suggesting the 
potential diagnostic relevance and therapeutic promise of targeting this unique tumor-regulating signaling axis in $\mathrm{PC}$ patients.

\section{Methods}

\subsection{Cell culture}

The PATU8988 PC cell line and the control HPDE6-C7 normal pancreatic ductal epithelial cell line (Procell,Wuhan,China) were cultured in DMEM containing $10 \% \mathrm{FBS}$ and penicillin/streptomycin in a $37^{\circ} \mathrm{C}$ $5 \% \mathrm{CO}_{2}$ incubator.

\subsection{Transfection}

Cells were transfected with miR-211-3p mimics or inhibitors through use of Lipofectamine 3000 (Invitrogen, Shanghai, China) based on provided directions. Cells were collected for downstream analyses at 24,48 , or $72 \mathrm{~h}$ post-transfection.

\section{3 qPCR}

Cells were cultured for $48 \mathrm{~h}$ in 6-well plates, after which total RNA was collected using Trizol and RNA purity was measured. ATP was diluted based on the amount of RNA used, after which the reverse transcription kit (Mei5 Biotechnology, China) was used based on provided directions to prepare a miRNA plus poly-A tail reaction system (total volume: $25 \mathrm{ul}$ ) in an enzyme-free reaction tube on ice. The reaction solution was mixed, centrifuged briefly at a low speed, and incubated for $15 \mathrm{~min}$ at $37^{\circ} \mathrm{C}$. A miRNA reaction was then prepared for cDNA synthesis ( $20 \mathrm{uL}$ volume) in enzyme-free reaction tubes on ice, with the reaction system being mixed, spun at a low speed, incubated for $50 \mathrm{~min}$ at $42^{\circ} \mathrm{C}$, and heated for $5 \mathrm{~min}$ at $85^{\circ} \mathrm{C}$. The resultant $\mathrm{CDNA}$ was stored at $-20^{\circ} \mathrm{C}$. For $\mathrm{qPCR}, 20 \mathrm{uL}$ reactions were prepared containing: 1 uL cDNA, $10 \mathrm{uL}$ ROX, $0.4 \mathrm{uL}$ of each primer $(10 \mathrm{uM})$, and $8.2 \mathrm{uL}$ of ddH2O. miRNA expression was detected with SYBR GREEN I. Relative gene expression was assessed via the $2^{-\triangle} \triangle \mathrm{CT}$ method as follows:

$\triangle \triangle \mathrm{CT}=\left(C \mathrm{~T}_{\text {target gene }}-\mathrm{C} \mathrm{T}_{\text {reference gene }}\right)$ in the experimental group-( $\left.\mathrm{CT}_{\text {target gene }}-\mathrm{CT}_{\text {reference gene }}\right)$ in the control group.The primer sequence and transfection gene sequence $\left(5^{\prime}-3^{\prime}\right)$ required for this experiment are shown(Table 1).

\subsection{CCK-8 analysis}

To assess cellular viability, cells were culture for 24,48 , or $72 \mathrm{~h}$ post-transfection, after which $10 \mathrm{uL}$ of CCK-8 reagent was added to each well for $2 \mathrm{~h}$ during which the reagent was reduced by the dehydrogenase activity of mitochondria in live cells. Absorption (OD) at $450 \mathrm{~nm}$ in each well was then assessed using a microplate reader.

\subsection{Migration assay}


After transfection cells were cultured to $80-90 \%$ confluence. They were then washed thrice with PBS, harvested using $0.25 \%$ trypsin-EDTA for $1 \mathrm{~min}$, and $1 \mathrm{~mL}$ of serum-free media was then added. A single cell suspension was then prepared, and Trypan blue was added to the suspension for 2 min at room temperature. Next, $10 \mathrm{uL}$ of these cells were counted using a hemocytometer. Cells were counted under low magnification (10x10 times), with cells being counted in the four corners of the hemocytometer field, after which cell number was measured as follows: cell number $/ \mathrm{ml}=(a+b+c+d) / 4 \times 10 \times 10^{4} / \mathrm{ml}$. Target cell number per counting field were between 20 and 300, with samples otherwise being diluted again and recounted. Next, 800 uL of culture media containing 10\% FBS was added to the upper chamber of a 24-well Transwell insert, after which $200 \mathrm{uL}$ of prepared cell suspensions from appropriate transfection and control groups were added per well $\left(4 \times 10^{3} /\right.$ well). Cells were incubated for $48 \mathrm{~h}$, after which the Transwell chamber was taken out, washed with PBS, fixed with $4 \%$ formaldehyde for $20 \mathrm{~min}$, and stained with $0.1 \%$ crystal violet staining solution for $30 \mathrm{~min}$. Background cells were removed by washing with PBS and gently swabbing with a cotton swab. Cells that migrated to the lower layer of the Transwell chamber were counted with an inverted microscope (10×20 times). Groups of cells were selected at random and counted in 5 fields of view per sample.

\subsection{Invasion assay}

Matrigel was thawed at $4^{\circ} \mathrm{C}$ overnight, and was then diluted 1:3 using serum-free media. The upper chamber of a 24-well Transwell insert was then coated with $40 \mathrm{uL}$ of diluted Matrigel and placed in an incubator for $2 \mathrm{~h}$ to allow the gel to solidify. After transfection, the cells in each group were cultured to 80$90 \%$ confluence, washed thrice with PBS, digested with $0.25 \%$ trypsin containing EDTA for about 1 min, and $1 \mathrm{ml}$ of serum-free medium was added to terminate the digestion. A single-cell suspension was prepared and cells were stained for 2 min with Trypan blue, after which $10 \mathrm{uL}$ of cells were assessed in a counting plate and quantified via microscopy. Next, $800 \mathrm{uL}$ of media containing $10 \%$ serum was added to the upper chamber of a 24-well Transwell insert, followed by the addition of $200 \mathrm{uL}$ of prepared cell suspensions ( $2 \times 10^{4}$ cells/well). After a $48 \mathrm{~h}$ incubation, chambers were removed, rinsed with PBS, fixed for 20 min with $4 \%$ formaldehyde, and stained for 30 min with $0.1 \%$ crystal violet. Background cells were removed by washing with PBS and gently swabbing with a cotton swab. Cells that invaded to the lower layer of the Transwell chamber were counted with an inverted microscope (10×20 times). Groups of cells were selected at random and counted in 5 fields of view per sample.

\subsection{Protein collection and preparation for Western blotting}

At $48 \mathrm{~h}$ post-transfection, cells in 6-well plates were washed thrice with chilled PBS, lysed with enhanced RIPA lysis buffer (Boster Company,Wuhan,China) containing a protease inhibitor (PMSF) (Boster Company), scraped, allowed to stand for $30 \mathrm{~min}$ on ice, vortexing every $10 \mathrm{~min}$, and spun for $15 \mathrm{~min}$ at $12,000 \mathrm{rpm}$ at $4^{\circ} \mathrm{C}$. Protein levels in supernatants were assessed via BCA assay. Protein amounts were then mixed with 4 uL of SDS-PAGE loading buffer (5X) (Boster Company), and the volume was increased to $20 \mathrm{uL}$ with RIPA lysis buffer. Samples were then denatured for $5 \mathrm{~min}$ at $95^{\circ} \mathrm{C}$, after which they were stored at $-20^{\circ} \mathrm{C}$. 


\subsection{Western blotting}

For protein separation, $10 \%$ SDS-PAGE gels were prepared with a $5 \%$ stacking gel region. Proteins were electrophoretically separated at $80 \mathrm{~V}$ until proteins entered the separation gel, at which time the voltage was increased to $120 \mathrm{~V}$. Proteins were then transferred onto PVDF membranes (Millipore, USA), which were blocked using $5 \%$ non-fat milk for $1 \mathrm{~h}$ with constant shaking ( $60 \mathrm{rpm})$ at room temperature, after which appropriate primary antibodies (1:1000, ABclonal, China) were added in primary antibody diluent (Boster Company, Wuhan, China), with polyclonal anti-GAPDH (Bioworld Company, USA) serving as a loading control. Blots were probed overnight at $4^{\circ} \mathrm{C}$, rinsed thrice with $1 \times$ TBST (100 rpm; $10 \mathrm{~min} /$ wash), and probed with HRP-labeled secondary antibodies (1:5000, Boster Company, Wuhan, China) for $1 \mathrm{~h}$ at room temperature with constant shaking $(60 \mathrm{rpm})$. Membranes were then developed using an ECL reagent and an imaging instrument, with the Image Lab software being used to detect protein bands.

\subsection{Analysis of cellular apoptosis}

At $48 \mathrm{~h}$ post-transfection, apoptotic cell death was assessed by dual staining cells with Annexin V-FITC and propidium iodide (PI) with an apoptosis detection kit (Meilun, Dalian, China) based on provided directions. A flow cytometer (Thermo Company, USA) was then used to assess apoptotic cell death, analyzing live, dead, early apoptotic, and late apoptotic cells.

\subsection{Statistical analysis}

SPSS 22.0 was used for all statistical analyses in this study. Data were averaged from three independent experiments. Data were compared between groups using one-way ANOVAs, and were compared within groups via LSD t-tests. $P<0.05$ was the threshold of statistical significance. GraphPad Prism 7.0 and Adobe Photoshop CC2019 were used to prepare Figures.

\section{Results}

While miR-221-3p has been reported to play an oncogenic role in many cancers, its specific functions in PC are poorly understood. As such, we began by exploring miR-221-3p expression levels in PC cell lines and the relationship between this miRNA and PC cell behavior in vitro. We then confirmed the ability of this miRNA to regulate PTEN and P53 in PC cells to influence tumor progression.

\subsection{Analyses of miR-221-3p knockdown and overexpression}

The $2^{-\triangle \Delta C T}$ method was used to assess relative gene expression, yielding the results shown in Table 1 and Figure 1.

\subsection{Analysis of PC cell proliferation}

Following miR-221-3p inhibition or overexpression in PATU8988 cells, a CCK-8 assay was performed to assess cell viability as discussed in the Methods section above (Figure 2). 


\subsection{Analysis of PC cell migration}

At $24 \mathrm{~h}$ post-transfection, cells were added to a Transwell insert at an appropriate density for $48 \mathrm{~h}$. They were then fixed, stained, and counted via microscopy (Figure 3 ).

\subsection{Analysis of PC cell invasion}

At $24 \mathrm{~h}$ post-transfection, cells were added to the Transwell chamber at an appropriate density and cultured for an additional $48 \mathrm{~h}$. Cells that had invaded through the Matrigel layer were then counted (Figure 4).

\subsection{Western blotting analysis}

Photoshop was used to process protein band images, with ImageJ being used for densitometric analyses (Figure 5).

\subsection{Analysis of PC cell apoptosis}

To evaluate the apoptotic death of PC cells, they were next subjected to Annexin V-FITC/PI staining and assessed via flow cytometry. The frequency of apoptotic PC cells rose significantly following miR-221-3p inhibition, and decreased upon miR-221-3p overexpression (Figure 6), suggesting that miR-221-3p can suppress the apoptotic death of PC cells.

\section{Discussion}

$\mathrm{PC}$ is among the deadliest cancers in the world, ${ }^{31}$ ranking $9^{\text {th }}$ and $10^{\text {th }}$ in terms of tumor incidence in the USA among men and women, respectively, in addition to being the 4th leading cause of tumor-related death as of 2021. ${ }^{32}$ Similarly, the National Cancer Center of China statistics from 2017 indicated that PC was 7 th and 11th in terms of tumor incidence in men and women, respectively, and was the 6th leading cause of tumor-related death. ${ }^{33} \mathrm{PC}$ patients still face a poor prognosis despite improvements in associated surgical and chemotherapeutic treatment options. ${ }^{34}$ One of the primary drivers of poor PC patient survival is that tumor invasion and distant metastasis often occur very early during the process of disease progression. ${ }^{35}$ As such, it is critical that PC be diagnosed at an early stage to improve patient treatment outcomes, emphasizing the need for novel approaches to diagnosing and treating this cancer type.

Through their ability to regulate diverse oncogenic processes, miRNAs can act as tumor suppressors or oncogenes. ${ }^{36,37}$ Virtually all cancer types have been found to exhibit miRNA dysregulation, ${ }^{38}$ and these miRNAs can control cellular proliferation, apoptosis, and growth. ${ }^{39}$ Specific miRNAs have been identified as novel biomarkers with relevance in the context of tumor diagnosis, prognostic evaluation, and treatment. ${ }^{40}$ MiR-221-3p is an oncogenic miRNA in liver, ${ }^{41}$ colorectal, ${ }^{42}$ breast, ${ }^{43}$ lung ${ }^{44}$ prostate ${ }^{45}$ and gastrointestinal stromal cancers, ${ }^{46}$ whereas it is a tumor suppressor in cholangiocarcinoma. ${ }^{47}$ There is 
strong evidence that miR-221-3p can regulate tumor cell proliferative, invasive, metastatic, chemoresistant, and apoptotic activity. ${ }^{48-50}$ Therefore, understanding the role of miR-221-3p in PC is of great value.

Herein, PATU8988 cells were used to establish models of miR-221-3p upregulation and downregulation as validated via qPCR. CCK-8 assays indicated that miR-221-3p overexpression enhanced proliferation, while Transwell-based assays further indicated that such overexpression enhanced PC cell migratory and invasive activity. Moreover, flow cytometry indicated that miR-221-3p overexpression reduced rates of PC cell apoptosis. The inhibition of this miRNA had the opposite effect.

To assess how miR-221-3p influenced downstream oncogenic pathways in PC, we herein performed a series of predictive bioinformatics analyses and related functional in vitro experiments. P53 is among the most studied tumor suppressors in mammalian cells, with the mutation or inactivation of P53 being a hallmark of a large percentage of human cancers. Mutated P53 can both lose its normal WT tumor suppressor activity and can even contribute to malignant disease progression in some cases. ${ }^{51}$ Suzuki et al. found that miRNA maturation is dependent on P53 regulation. ${ }^{52}$ The MDM2 gene is upregulated by P53 and binds to its inactivation domain, disrupting the recruitment of other transcriptional regulators necessary for gene expression. ${ }^{53}$ MDM2 can also serve as an E3 ubiquitin ligase to promote P53 degradation. ${ }^{54}$ Through this mechanism, P53 and MDM2 regulate one another in a feedback loop such that $\mathrm{P} 53$ promotes MDM2 upregulation which in turn promotes $\mathrm{P} 53$ downregulation. ${ }^{55}$ Cyclin $\mathrm{G} 1 \mathrm{can}$ additionally serve as a negative feedback regulator that decreases P53 stability and promotes protein degradation. ${ }^{56}$ PTEN is a central tumor suppressor gene with documented roles as a regulator of cellular apoptosis, proliferation, and growth. ${ }^{57}$ PTEN is encoded on chromosome 10q23.3, and suppresses oncogenesis via its dual lipid and protein phosphatase activity. ${ }^{58-60}$ Many malignant tumors exhibit PTEN inactivation and consequent overactivation of $A K T$, in turn driving enhanced invasion and radioresistance together with a decrease in apoptotic susceptibility. ${ }^{61-63}$ PTEN regulates tumor cell survival, proliferation, and metastatic progression. ${ }^{64,65}$ Several miRNAs including miR-21 and miR-214 are known PTEN regulators. ${ }^{66-70}$ Yang et al. ${ }^{71}$ posited that miR-221-3p was able to promote Capan2 PC cell proliferation via the PTEN/AKT axis. Consistently, we herein found that P53 and PTEN protein levels were reduced in PC cells following miR-221-3p overexpression and increases when this miRNA was inhibited, consistent with the ability of this miRNA to target these two genes. However, further work will be necessary to clarify the related signaling mechanisms whereby miR-221-3p regulates these two tumor suppressor genes. However, this study only evaluated this miRNA at the cellular level, and as such, our future research will focus on examining the diagnostic and prognostic relevance of miR-221-3p in clinical settings. In conclusion, miR-221-3p overexpression within PC cells can enhance tumor progression and malignancy.Further work is needed to explore the mechanisms whereby this miRNA can shape the pathogenesis of PC in order to highlight novel approaches to diagnosing and treating PC.

\section{Conclusion}


The upregulation of miR-221-3p in PATU8988 cells enhanced their proliferative, migratory, and invasive activity while suppressing PC Cell apoptosis. In contrast, the downregulation of this miRNA had the opposite effect. Mechanistically, miR-221-3p was able to target P53 and PTEN as a means of regulating PC progression. Together, these data may highlight a novel approach to exploring the pathogenesis of PC in order to provide patients with novel therapeutic modalities capable of improving survival rates and overall quality of life.

\section{Declarations}

\section{Acknowledgment}

Not applicable.

\section{Author Contributions}

JJW and ZHS conceived and designed the study and drafted the manuscript. JJW,MG,ZHY,FGR and SCB collected, analyzed, and interpreted the experimental data.JJW and ZHS revised the manuscript for important intellectual content. All authors read and approved the final manuscript.

\section{Funding}

This study is funded by the Natural Science Foundation of Shanxi Province(201801D121217).

\section{Availability of data and materials}

The datasets used and/or analyzed during the current study are available from the corresponding author on reasonable request.

\section{Ethics approval and consent to participate}

This study is approved by the ethics committee of the Second Hospital of Shanxi Medicine University.

\section{Consent for publication}

Not applicable.

\section{Competing interests}

The authors declare that they have no competing interests.

\section{References}

1. Siegel R, Ma J, Zou Z, Ahmedin M. Cancer statistics, 2014. CA Cancer J Clin. 2014;64:9-14.

2. Gupta GP, Massague J. Cancer metastasis:building a framework. Cell. 2006;127(4):679-695. 
3. Chan B, Manley J, Lee J, Singh SR. The emerging roles of microRNAs in cancer metabolism. Cancer Lett. 2015;356(2 Pt A):301-308.

4. Chitkara D, Mittal A, Mahato RI. miRNAs in pancreatic cancer:therapeutic potential,delivery challenges and strategies. Adv Drug Deliv Rev. 2015;81:34-52.

5. Berindan-Neagoe I, Monroig Pdel C, Pasculli B, Calin GA. MicroRNAome genome:a treasure for cancer diagnosis and therapy. CA Cancer J Clin. 2014;64(5):311-336.

6. Ali S, Suresh R, Banerjee S, et al. Contribution of microRNAs in understanding the pancreatic tumor microenvironment involving cancer associated stellate and fibroblast cells. Am J Cancer Res. 2015;5(3):1251-1264.

7. GaRoFalo M, QuiNtavalle C, RomaNo G, cRoce CM, coNDoRelli G. miR221/222 in cancer: their role in tumor progression and response to therapy. Curr Mol Med. 2012;12:27-33.

8. Zhang $X$, Shao S, Geng H, et al. Expression profiles of six circulating microRNAs critical to atherosclerosis in patients with subclinical hypothyroidism: a clinical study. J Clin Endocrinol Metab. 2014;99:766-774.

9. Xue Y, Wei Z, Ding H, et al. MicroRNA-19b/221/222 induces endothelial cell dysfunction via suppression of PGC-1a in the progression of atherosclerosis. Atherosclerosis. 2015;241:671-681.

10. Ergun S, Tayeb TS, Arslan A, et al. The investigation of miR-221-3p and PAK1 gene expressions in breast cancer cell lines. Gene. 2015;25:377-381.

11. Tao K, Yang J, Guo Z, et al. Prognostic value of miR-221-3p, miR-342-3p and miR-491-5p expression in colon cancer. Am J Transl Res. 2014;18:391-401.

12. Wu Q, Ren $X$, Zhang $Y$, et al. MiR-221-3p targets ARF4 and inhibits the proliferation and migration of epithelial ovarian cancer cells. Biochem Biophys Res Commun. 2018;497(4):1162-1170.

13. Shi J, Zhang Y, Jin N, Li Y, Wu S, Xu L. MicroRNA-221-3p plays an oncogenic role in gastric carcinoma by inhibiting PTEN expression. Oncol Res. 2017 Apr;25(4):523-536.

14. Coskunpinar E, Cakmak HA, Kalkan AK, Tiryakioglu NO, Erturk M, Ongen Z. Circulating miR-221-3p as a novel marker for early prediction of acute myocardial infarction. Gene. 2016;591(1):90-96.

15. Kristensen $\mathrm{H}$, Thomsen AR, Haldrup $\mathrm{C}$, et al. Novel diagnostic and prognostic classifiers for prostate cancer identified by genome-wide microRNA profiling. Oncotarget. 2016;7(21):30760.

16. Li F, Xu JW, Wang L, Liu H, Yan Y, Hu SY. MicroRNA-221-3p is up-regulated and serves as a potential biomarker in pancreatic cancer. Artif Cells Nanomed Biotechnol. 2018;46(3):482-487.

17. Sarkar S, Dubaybo H, Ali S, et al. Down-regulation of miR-221 inhibits proliferation of pancreatic cancer cells through up-regulation of PTEN, p27(kip1), p57(kip2), and PUMA. Am J Cancer Res. 2013;3(5):465-477.

18. Deng L, Lei Q, Wang Y, et al. Downregulation of miR-221-3p and upregulation of its target gene PARP1 are prognostic biomarkers for triple negative breast cancer patients and associated with poor prognosis. Oncotarget. 2017;8(65):108712-108725. 
19. Handa H, Murakami Y, Ishihara R, Kimura-Masuda K, Masuda Y. The Role and Function of microRNA in the Pathogenesis of Multiple Myeloma. Cancers. 2019;11(11).

20. Vogelstein B, Lane D, Levine A. Surfing the P53 network. Nature. 2000;408:307-310.

21. Zhang MF, Zhang ZY, Fu J, Yang YF, Yun JP. Correlation between expression of p53, p21/WAF1, and MDM2 proteins and their prognostic significance in primary hepatocellular carcinoma. $J$ Trans/ Med. 2009;7:110.

22. Kim D, Song J, Jin EJ. Withdrawal: MicroRNA-221 regulates chondrogenic differentiation through promoting proteosomal degradation of Slug by targeting Mdm2. J Biol Chem. 2020;295(2):668.

23. Li J, Yen C, Liaw D, et al. PTEN, a putative protein tyrosine phosphatase gene mutated in human brain, breast, and prostate cancer. Science. 1997;275(5308):1943.

24. Teng DH, Hu R, et al. MMAC1/PTEN mutations in primary tumor specimens and tumor cell lines. Cancer Res. 1997;57(23):5221-5225.

25. Sun Y, St Clair DK, Fang F, et al. The radiosensitization effect of parthenolide in prostate cancer cells is mediated by nuclear factor-kappaB inhibition and enhanced by the presence of PTEN. Mol Cancer Therap. 2007;6(9):2477-2486.

26. Jiang Z, Pore N, Cerniglia GJ, et al. Phosphatase and tensin homologue deficiency in glioblastoma confers resistance to radiation and temozolomide that is reversed by the protease inhibitor nelfinavir. Cancer Res. 2007;67(9):4467-4473.

27. Lange AM, Lo HW. Inhibiting TRK Proteins in Clinical Cancer Therapy. Cancers. 2018;10(4).

28. Ge H, Cao YY, Chen LQ, et al. PTEN polymorphisms and the risk of esophageal carcinoma and gastric cardiac carcinoma in a high incidence region of China. Dis Esophagus. 2008;21(5):409-415.

29. Cinti C, Vindigni C, Zamparelli A, et al. Activated Akt as an indicator of prognosis in gastric cancer. Virchows Archiv. 2008;453(5):449-455.

30. Ihle MA, Trautmann $\mathrm{M}$, Kuenstlinger $\mathrm{H}$, et al. miRNA-221 and miRNA-222 induce apoptosis via the KIT/AKT signalling pathway in gastrointestinal stromal tumours. Mol Oncol. 2015;9(7):1421-1433.

31. Siegel R, Ma J, Zou Z, Ahmedin M. Cancer statistics, 2014. CA Cancer J Clin. 2014;64:9-

32. Siegel RL, Miller KD, Fuchs HE, Jemal A. Cancer Statistics, 2021. CA. 2021;71(1):7-33.

33. Chen X, Yi B, Liu Z, et al. Global, regional and national burden of pancreatic cancer, 1990 to 2017: Results from the Global Burden of Disease Study 2017. Pancreatology. 2020;20(3):462-469.

34. Vincent A, Herman J, Schulick R, Hruban RH, Goggins M. Pancreatic cancer. Lancet. 2011;378(9791):607-620.

35. Von Hoff DD, Ramanathan RK, Borad MJ, et al. Gemcitabine plus nab-paclitaxel is an active regimen in patients with advanced pancreatic cancer: a phase I/II trial. J Clin Oncol. 2011;29(34):4548-4554.

36. Esteller M. Non-coding RNAs in human disease. Nat Rev Genet. 2011;12(12):861-874.

37. Garzon R, Calin GA, Croce CM. MicroRNAs in Cancer. Ann Rev Med. 2009;60:167-179.

38. Roberts TC, Wood MJ. Therapeutic targeting of non-coding RNAs. Essays Biochem. 2013;54:127145. 
39. Gebert LFR, MacRae IJ. Regulation of microRNA function in animals. Nat Rev Mol cell Boil. 2019;20(1):21-37.

40. White NM, Fatoohi E, Metias M, Jung K, Stephan C, Yousef GM. Metastamirs: a stepping stone towards improved cancer management. Na Rev Clin Oncol. 2011;8(2):75-84.

41. Bae HJ, Jung KH, Eun JW, et al. MicroRNA-221 governs tumor suppressor HDAC6 to potentiate malignant progression of liver cancer. $J$ hepatol. 2015;63(2):408-419.

42. Liu S, Sun X, Wang M, et al. A microRNA 221- and 222-mediated feedback loop maintains constitutive activation of NFKB and STAT3 in colorectal cancer cells. Gastroenterology. 2014;147(4):847-859.

43. Shah MY, Calin GA. MicroRNAs miR-221 and miR-222: a new level of regulation in aggressive breast cancer. Genome Med. 2011;3(8):56.

44. Garofalo M, Di Leva G, Romano G, et al. miR-221\&222 regulate TRAlL resistance and enhance tumorigenicity through PTEN and TIMP3 downregulation. Cancer Cell. 2009;16(6):498-509.

45. Galardi S, Mercatelli N, Farace MG, Ciafrè SA. NF-kB and c-Jun induce the expression of the oncogenic miR-221 and miR-222 in prostate carcinoma and glioblastoma cells. Nucleic Acids Res. 2011;39(9):3892-3902.

46. Gits CM, van Kuijk PF, Jonkers MB, et al. MiR-17-92 and miR-221/222 cluster members target KIT and ETV1 in human gastrointestinal stromal tumours. Br J Cancer. 2013;109(6):1625-1635.

47. Okamoto K, Miyoshi K, Murawaki Y. miR-29b, miR-205 and miR-221 enhance chemosensitivity to gemcitabine in HuH28 human cholangiocarcinoma cells. PloS One. 2013;8(10):e77623.

48. Ciafrè SA, Galardi S, Mangiola A, et al. Extensive modulation of a set of microRNAs in primary glioblastoma. Biochem Biophy Res Commun. 2005;334(4):1351-1358.

49. Besson A, Gurian-West M, Schmidt A, Hall A, Roberts JM. p27Kip1 modulates cell migration through the regulation of RhoA activation. Genes Dev. 2004;18(8):862-876.

50. Nikiforova MN, Tseng GC, Steward D, Diorio D, Nikiforov YE. MicroRNA expression profiling of thyroid tumors: biological significance and diagnostic utility. J Clin Endocrinol Metab. 2008;93(5):16001608.

51. Muller PA, Vousden KH. Mutant p53 in cancer: new functions and therapeutic opportunities. Cancer Cell. 2014;25(3):304-317.

52. Suzuki HI, Yamagata K, Sugimoto K, Iwamoto T, Kato S, Miyazono K. Modulation of microRNA processing by p53. Nature. 2009;460(7254):529-533.

53. Oliner JD, Pietenpol JA, Thiagalingam S, Gyuris J, Kinzler KW, Vogelstein B. Oncoprotein MDM2 conceals the activation domain of tumour suppressor p53. Nature. 1993;362(6423):857-860.

54. Brooks CL, Gu W. p53 ubiquitination: Mdm2 and beyond. Mol Cell. 2006;21(3):307-315.

55. Wu X, Bayle JH, Olson D, Levine AJ. The p53-mdm-2 autoregulatory feedback loop. Genes Dev. 1993;7(7a):1126-1132. 
56. Ohtsuka T, Ryu H, Minamishima YA, Ryo A, Lee SW. Modulation of p53 and p73 levels by cyclin G: implication of a negative feedback regulation. Oncogene. 2003;22(11):1678-1687.

57. Ge H, Cao YY, Chen LQ, et al. PTEN polymorphisms and the risk of esophageal carcinoma and gastric cardiac carcinoma in a high incidence region of China. Dis Esophagus 2008;21(5):409-415.

58. Li J, Yen C, Liaw D, et al. PTEN, a putative protein tyrosine phosphatase gene mutated in human brain, breast, and prostate cancer. Science. 1997;275(5308):1943-1947.

59. Steck PA, Pershouse MA, Jasser SA, et al. Identification of a candidate tumour suppressor gene, MMAC1, at chromosome 10q23.3 that is mutated in multiple advanced cancers. Nat Genet. 1997;15(4):356-362.

60. Li DM, Sun H. TEP1, encoded by a candidate tumor suppressor locus, is a novel protein tyrosine phosphatase regulated by transforming growth factor beta. Cancer Res. 1997;57(11):2124-2129.

61. Ge H, Cao YY, Chen LQ, et al. PTEN polymorphisms and the risk of esophageal carcinoma and gastric cardiac carcinoma in a high incidence region of China. Dis Esophagus. 2008;21(5):409-415.

62. Cinti C, Vindigni C, Zamparelli A, et al. Activated Akt as an indicator of prognosis in gastric cancer. Virchows Archiv. 2008;453(5):449-455.

63. Pappas G, Zumstein LA, Munshi A, Hobbs M, Meyn RE. Adenoviral-mediated PTEN expression radiosensitizes non-small cell lung cancer cells by suppressing DNA repair capacity. Cancer Gene Ther. 2007;14(6):543-549.

64. Zhang H, Xu HL, Wang YC, Lu ZY, Yu XF, Sui DY. 20(S)-Protopanaxadiol-Induced Apoptosis in MCF-7 Breast Cancer Cell Line through the Inhibition of PI3K/AKT/mTOR Signaling Pathway. Int J Mol Sci. 2018; 19(4).

65. Mardente S, Mari E, Massimi I, et al. HMGB1-Induced Cross Talk between PTEN and miRs 221/222 in Thyroid Cancer. BioMed Res Int. 2015;2015:512027.

66. Wickramasinghe NS, Manavalan TT, Dougherty SM, Riggs KA, Li Y, Klinge CM. Estradiol downregulates miR-21 expression and increases miR-21 target gene expression in MCF-7 breast cancer cells. Nucleic Acids Res. 2009;37(8):2584-2595.

67. Qi L, Bart J, Tan LP, et al. Expression of miR-21 and its targets (PTEN, PDCD4, TM1) in flat epithelial atypia of the breast in relation to ductal carcinoma in situ and invasive carcinoma. BMC Cancer. 2009;9:163.

68. Talotta F, Cimmino A, Matarazzo MR, et al. An autoregulatory loop mediated by miR-21 and PDCD4 controls the AP-1 activity in RAS transformation. Oncogene. 2009;28(1):73-84.

69. Vinciguerra M, Sgroi A, Veyrat-Durebex C, Rubbia-Brandt L, Buhler LH, Foti M. Unsaturated fatty acids inhibit the expression of tumor suppressor phosphatase and tensin homolog (PTEN) via microRNA21 up-regulation in hepatocytes. Hepatology. 2009;49(4):1176-1184.

70. Yang $\mathrm{H}$, Kong $\mathrm{W}, \mathrm{He} \mathrm{L}$, et al. MicroRNA expression profiling in human ovarian cancer: miR-214 induces cell survival and cisplatin resistance by targeting PTEN. Cancer Res. 2008;68(2):425-433. 
71. Yang W, Yang Y, Xia L, et al. MiR-221 Promotes Capan-2 Pancreatic Ductal Adenocarcinoma Cells Proliferation by Targeting PTEN-Akt. Cell Physiol Biochem. 2016;38(6):2366-2374.

\section{Tables}

Table 1. Primer sequence and transfection gene sequence ( $\left.5^{\prime}-3^{\prime}\right)$ required for this experiment.

\begin{tabular}{ll}
\hline Gene and primer name & Primer sequence (5'-3') \\
\hline miR-221-3p mimics NC & 5'-UUCUCCGAACGUGUCACGUTT-3' \\
miR-221-3p mimics & 5'-AGCUACAUUGUCUGCUGGGUUUC-3' \\
miR-221-3p inhibitor NC & 5'-UUGUACUACACAAAAGUACUG-3' \\
miR-221-3p inhibitor & 5'-GAAACCCAGCAGACAAUGUAGCU-3' \\
miR-221-3p Forward Primer & 5'-AGCTACATTGTCTGCTGGGTTTC-3' \\
miR-221-3p Reverse Primer & 5'-GCAGGGTCCGAGGTATTC-3' \\
U6 Forward Primer & 5'-GCTTCGGCAGCACATATACT-3' \\
U6 Reverse Primer & 5'-GCAGGGTCCGAGGTATTC-3'
\end{tabular}

\section{Figures}




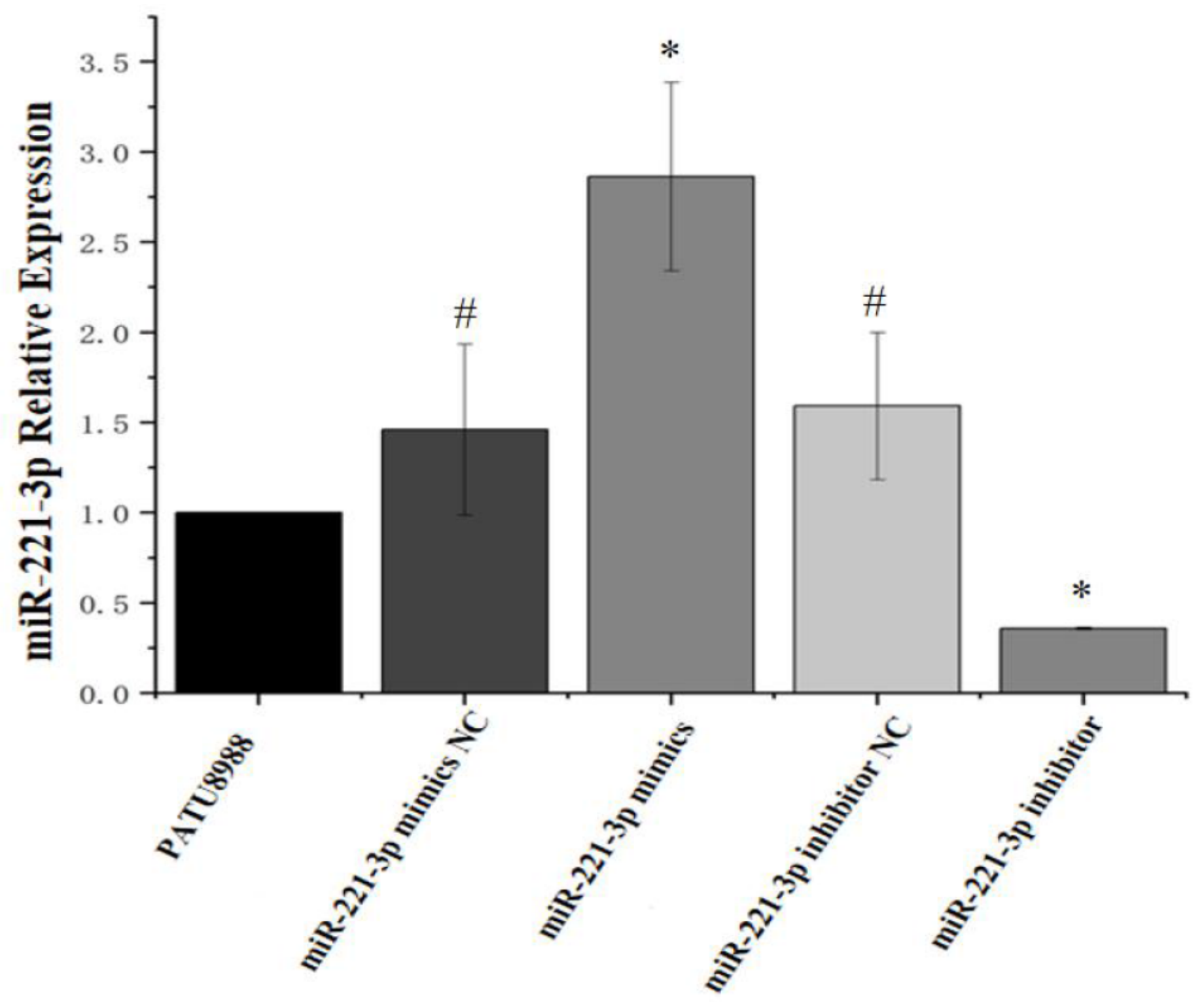

Figure 1

Figure 1

Relative to the control group, at $24 \mathrm{~h}$ post-transfection, PATU8988 cells transfected with miR-221-3p mimics NC or miR-221-3p inhibitor NC did not exhibit any alterations in miR-221-3p expression ( $\# \mathrm{P}>0.05)$, while miR-221-3p mimic transfection increased the expression of this miRNA $\left({ }^{*} \mathrm{P}<0.05\right)$, after miR-221-3p inhibitor transfection downregulated this miRNA ( $\left.{ }^{*}<<0.05\right)$. 


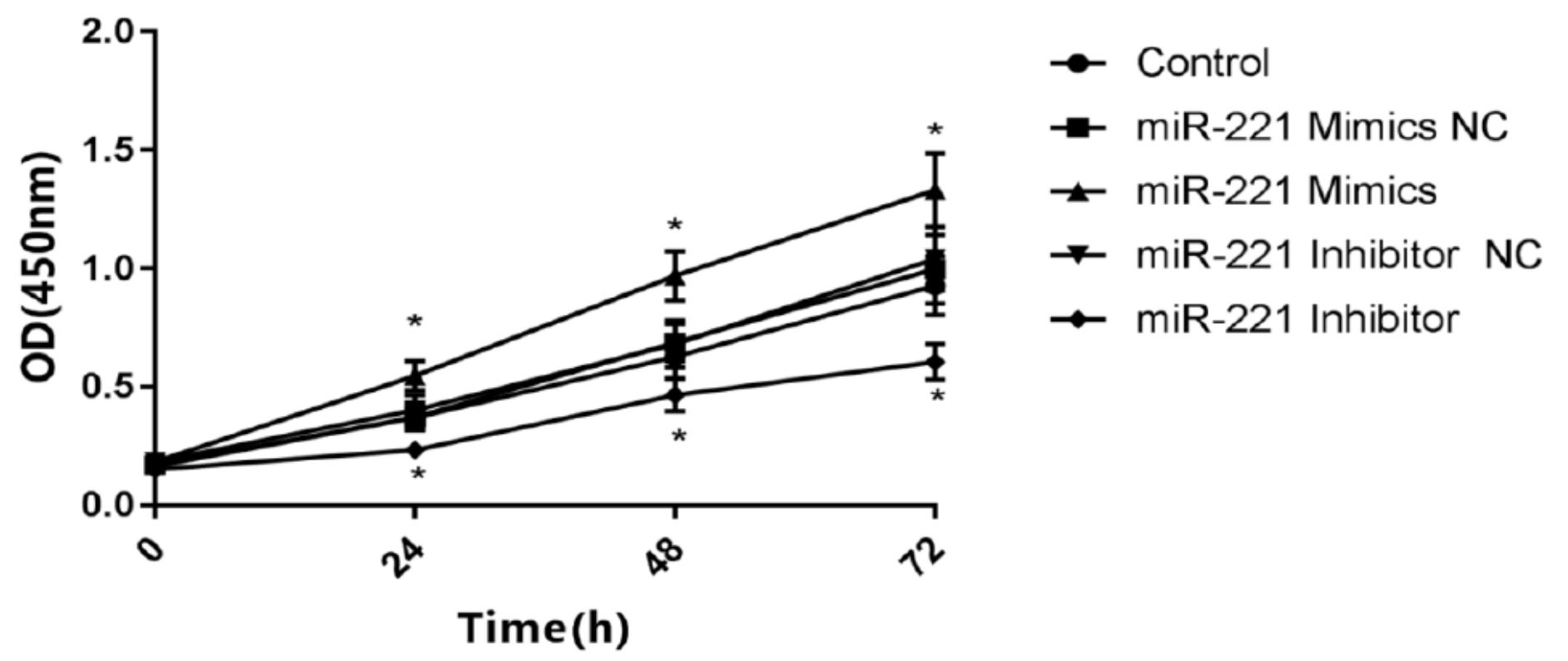

\section{Figure 2}

Figure 2

The absorbance (OD) value at $450 \mathrm{~nm}$ was assessed in the indicated treatment groups at 24,48 , and 72 $h$ post-transfection. The OD value for the miR-221-3p mimic group was significantly higher than that in the control and miR-221-3p mimic groups $(P<0.01)$, while the OD value in the miR-221-3p inhibitor group was significantly lower than that in the control and miR-221-3p inhibitor NC groups $(P<0.01)$. There were no significant differences among the three control groups with respect to the OD450 value $(P>0.05)$. 
Control
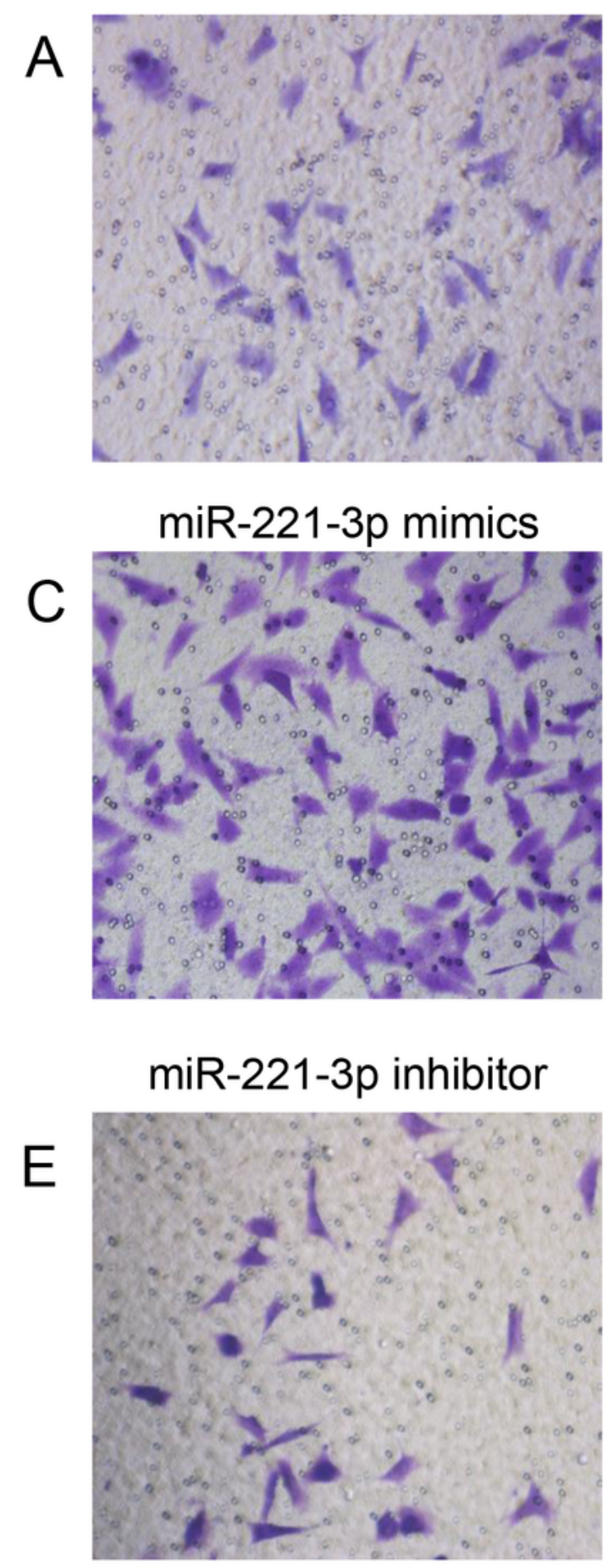

\section{miR-221-3p mimics NC}

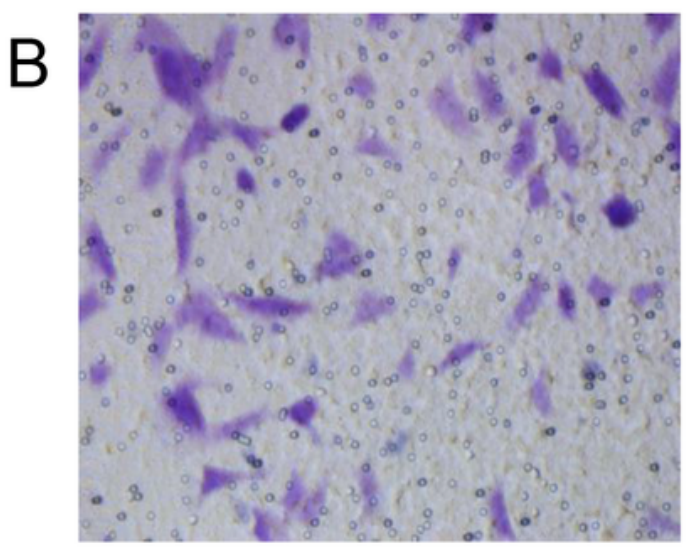

\section{miR-221-3p inhibitor NC}

$\mathrm{D}$

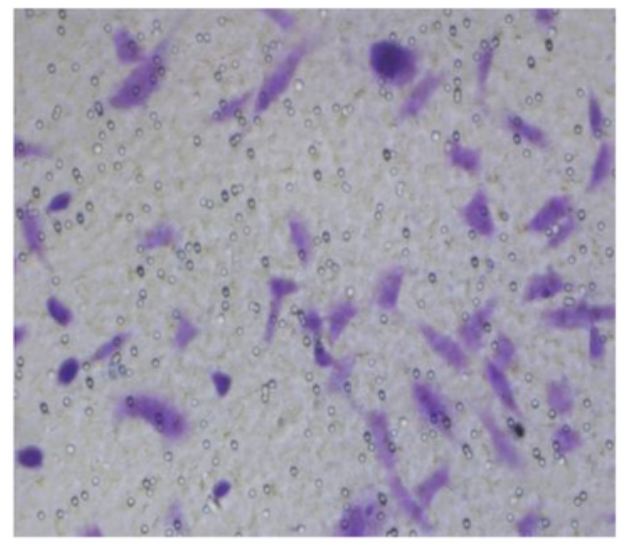

$\mathrm{F}$

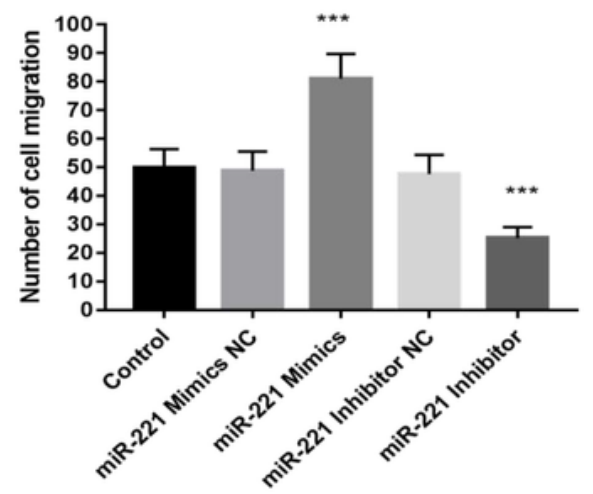

\section{Figure 3}

(A-E) Representative images of cells in the indicated treatment groups. $(F)$ The number of migratory cells in the miR-221-3p mimic group was significantly higher than that in the control and miR-221-3p mimics NC group $(P<0.01)$, while the number of migratory cells in the miR-221-3p inhibitor group was significantly lower than that in the control and miR-221-3p inhibitor NC groups $(P<0.01)$. There were no differences in cellular invasion among the three control groups $(P>0.05)$. 

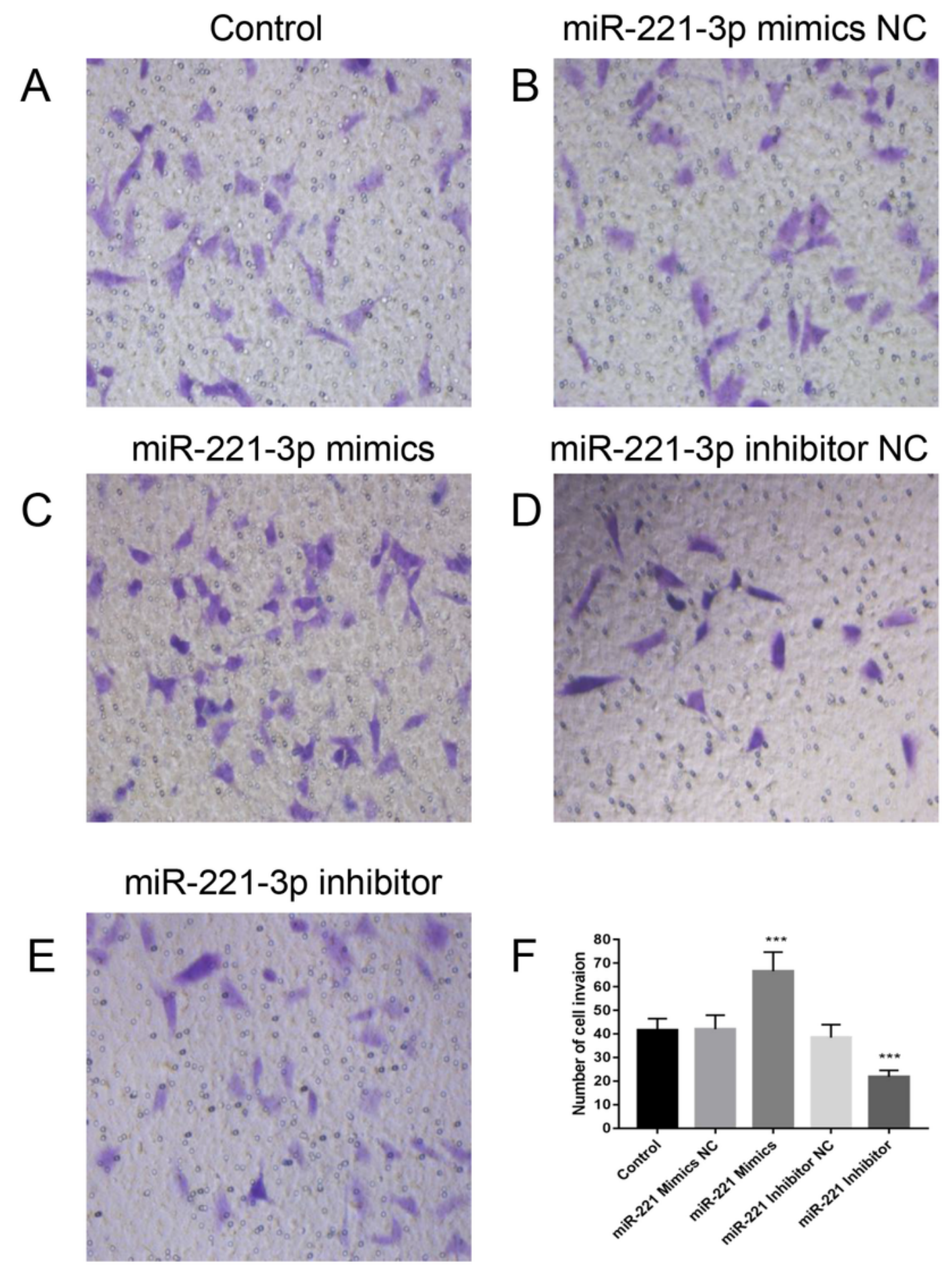

\section{Figure 4}

(A-E) Representative images of cells in the indicated treatment groups. $(F)$ The number of invasive cells in the miR-221-3p mimic group was significantly higher than that in the control and miR-221-3p mimics NC group $(P<0.01)$, while the number of invasive cells in the miR-221-3p inhibitor group was significantly reduced relative to the control and miR-221-3p inhibitor NC groups $(P<0.01)$. There were no differences in cellular invasion among the three control groups $(P>0.05)$. 


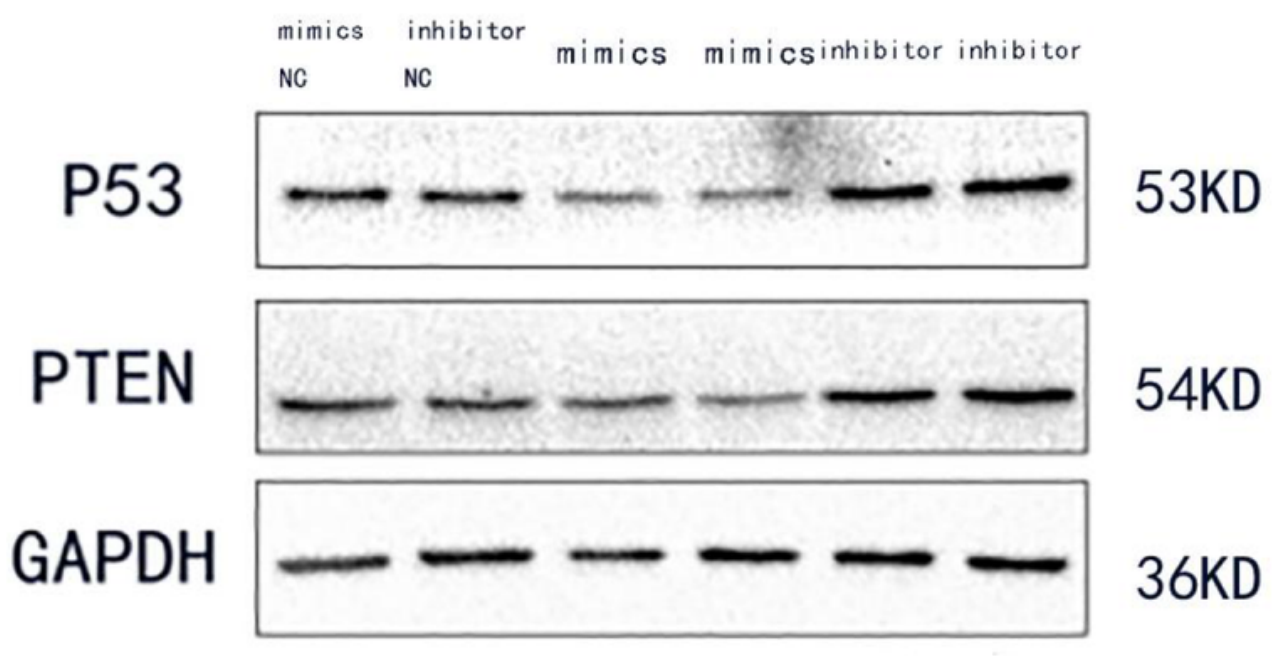

(A)
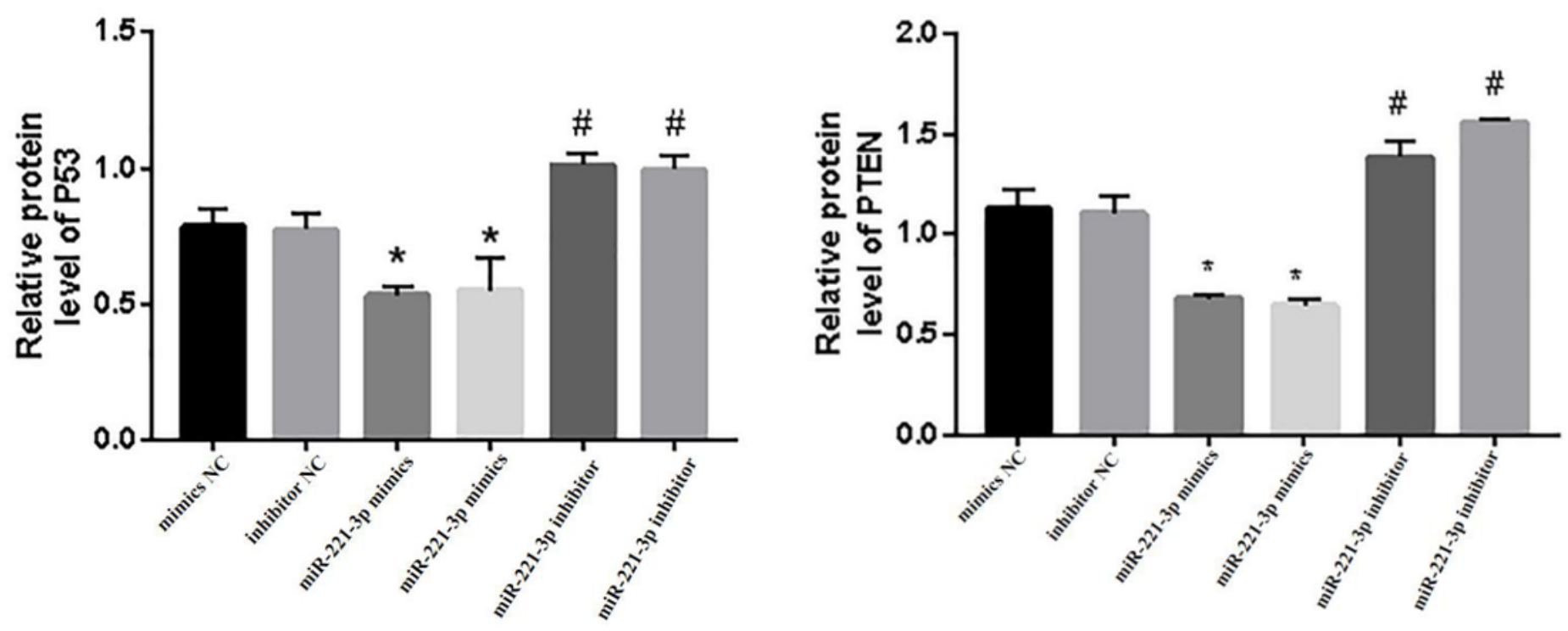

(B)

Figure 5

Figure 5

(A) P53, PTEN, and GAPDH protein levels were measured in the indicated groups. (B) At 24 h posttransfection, P53 and PTEN protein levels were measured by Western blotting, revealing no differences in these levels when comparing the NC and miR-221-3p inhibitor NC groups ( $P>0.05)$. P53 and PTEN levels were significantly reduced in the miR-221-3p mimic group as compared to the mimic NC group $\left({ }^{\star} P<0.05\right)$. 
Relative to the inhibitor NC group, P53 and PTEN protein levels were significantly increased in the miR221-3p inhibitor group $(\# \mathrm{P}<0.05)$.
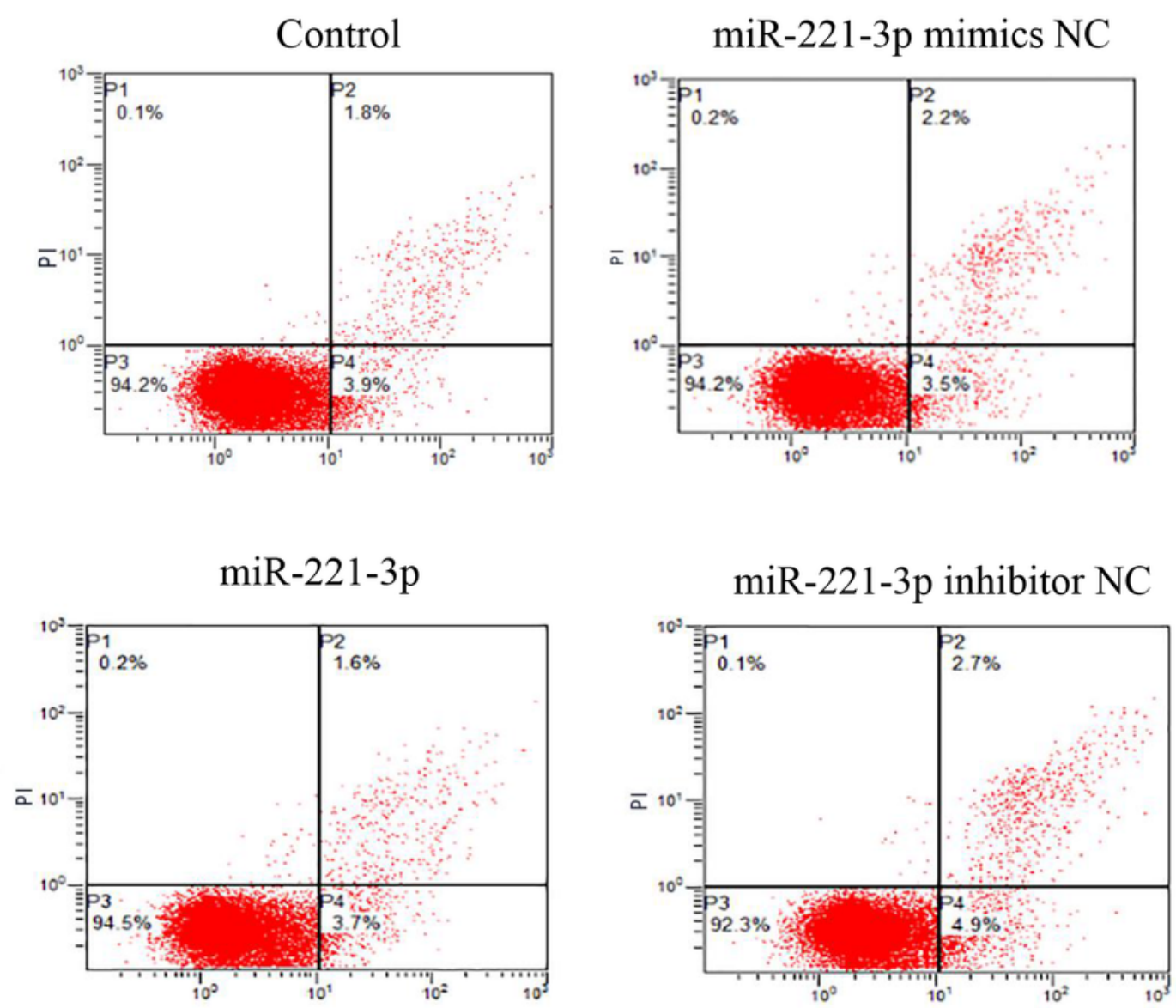

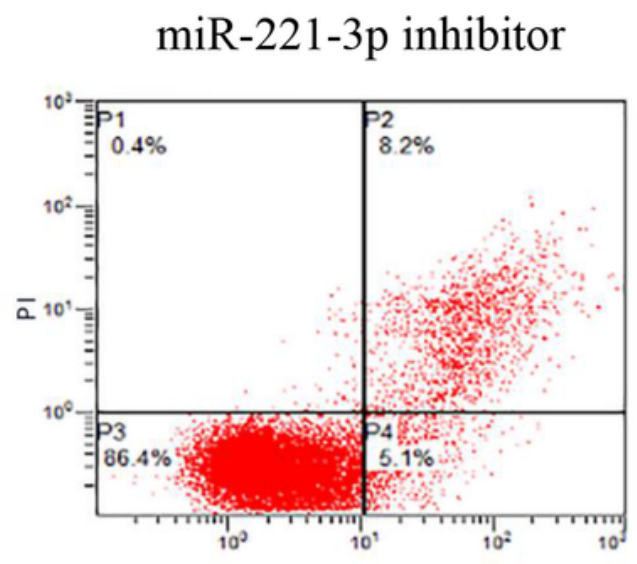

(A)

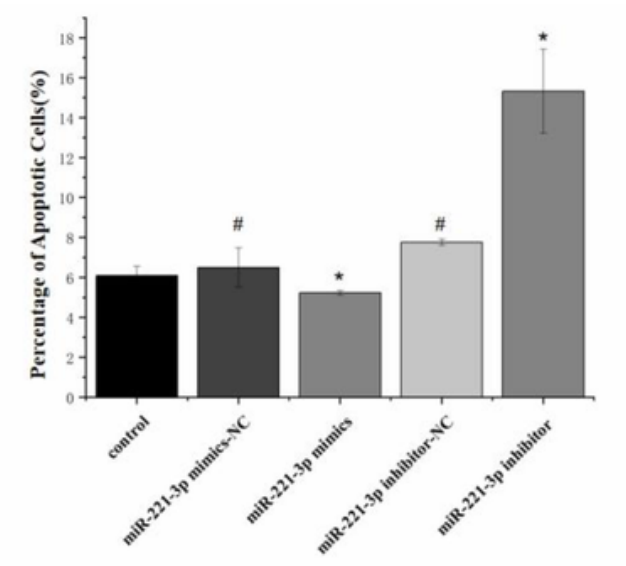

(B)

Figure 6

Figure 6

(A) Apoptotic induction in the indicated treatment groups. (B) Rates of apoptosis were significantly increased in the miR-221-3p inhibitor group relative to the control and the miR-221-3p inhibitor NC groups, 
while miR-221-3p mimic transfection significantly reduced the rate of apoptotic induction relative to control treatment. $\# \mathrm{P}>0.05,{ }^{*} \mathrm{P}<0.05$. 